\title{
Transatlantica
}

Revue d'études américaines. American Studies Journal

$1 \mid 2012$

Le roman policier, littérature transatlantique / Maisons

Hantées

\section{Paule Lévy, American Pastoral. La Vie réinventée, Paris, CNED/P.U.F., 2012}

\section{Anne-Laure Tissut}

\section{(2) OpenEdition}

\section{Journals}

Édition électronique

URL : https://journals.openedition.org/transatlantica/5706

DOI : $10.4000 /$ transatlantica. 5706

ISSN : 1765-2766

Éditeur

Association française d'Etudes Américaines (AFEA)

Référence électronique

Anne-Laure Tissut, «Paule Lévy, American Pastoral. La Vie réinventée, Paris, CNED/P.U.F., 2012 »,

Transatlantica [En ligne], 1 | 2012, mis en ligne le 06 janvier 2013, consulté le 04 février 2023. URL

http://journals.openedition.org/transatlantica/5706 ; DOI : https://doi.org/10.4000/transatlantica. 5706

Ce document a été généré automatiquement le 4 février 2023.

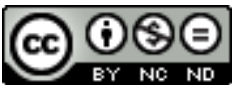

Creative Commons - Attribution - Pas d'Utilisation Commerciale - Pas de Modification 4.0 International - CC BY-NC-ND 4.0

https://creativecommons.org/licenses/by-nc-nd/4.0/ 


\title{
Paule Lévy, American Pastoral. La Vie réinventée, Paris, CNED/P.U.F., 2012
}

\author{
Anne-Laure Tissut
}

1 Paule Lévy, spécialiste de littérature américaine $\mathrm{du} \mathrm{xx}^{\mathrm{e}}$ siècle, et notamment de littérature juive américaine, auteur de l'ouvrage intitulé Figures de l'artiste. Identité et écriture dans la littérature juive américaine de la deuxième moitié $d u x^{e}$ siècle (Presses Universitaires de Bordeaux, 2007) et coordinatrice de Lectures de Philip Roth. American Pastoral (Presses Universitaires de Rennes, 2011), a su dans le présent livre faire découvrir d'autres facettes de American Pastoral et en proposer des analyses inédites, étayées par des citations encore peu ou pas exploitées par la critique.

2 Après une biographie sélective, qui opère une mise en scène pertinente de l'auteur de American Pastoral, Paule Lévy se livre à un panorama de l'œuvre de Philip Roth, détaillé mais uniformément orienté par le souci d'apporter un éclairage approprié sur le roman précis dont il est question dans l'ouvrage. Les structures de American Pastoral sont d'abord étudiées (29), ensuite la réception contrastée que lui réserve le lectorat (31), puis la pastorale, «métaphore structurante » (34) de ce roman, mythe auquel Roth se réfère "à des fins purement ironiques" (36). Le personnage central est ensuite analysé : le Suédois, incarnation du rêve américain dans ses deux aspects, matérialiste et pastoral, et surtout de la bonne conscience des classes moyennes américaines, cible principale de Roth dans la trilogie qu'ouvre American Pastoral (1997), et complétée par I Married a Communist (1998) puis The Human Stain (2000). Paule Lévy rappelle que destinée du personnage et destinée nationale sont étroitement liées dans ce roman, pour mieux souligner la spécificité de la pastorale proposée par Roth, qui ne signale " pas une dissidence mais au contraire un parfait 'isomorphisme avec le monde Wasp' $(A P, 89) »(40)$, une « absolue cohésion du moi » (40). L'adéquation parfaite de l'individu au pays est emblématisée par le gant, dont la symbolique multiforme est analysée page 40 : le héros "fait corps avec son métier ", et avec son pays, lit-on dans la sous-partie intitulée «Le mythe incarné, l'Amérique dans la peau » (37), formule dont la pertinence subtile et la grande force évocatrice sont à la hauteur des autres titres de parties et sous-parties. En l'occurrence, ce titre reflète l'ironie dont tout le roman est marqué, 
comme si le critique s'inspirait du style de Roth ou en subissait l'insidieuse contamination.

3 Tout au long du roman, Paule Lévy le souligne, l'ironie dramatique (41), le jeu sur les points de vue et les voix accordent au lecteur le privilège d'un point de vue complice, reposant sur la conscience de l'absurdité du choix intenable qu'a fait le Suédois, « choix de vie pour lequel Levov est en fait aussi peu culturellement disposé que l'était le Herzog de Saul Bellow» (41). Paule Lévy repère aussi le tissage d'un réseau métaphorique à partir de l'image de la surface et des masques, qui très tôt alerte le lecteur: "With the Swede indomitable on the playing field, the meaningless surface of life provided a bizarre, delusionary kind of substance $(A P, 4)$ ». L'auteure, on le voit, a le don d'orienter l'attention du lecteur sur certaines phrases discrètes, estompées par d'autres plus théâtrales ou rhétoriques, plus directement repérables et analysables : par exemple, la citation précédente, donnant au Suédois une fonction divertissante, soulignant l'effet d'aveuglement que peut suggérer sa blondeur symbolique.

Paule Lévy analyse le jeu des masques et des surfaces à tous niveaux, entre autres celui de l'attentat de Merry, qui selon Jerry vise la «façade de mensonges " érigée par son père, ou celui de la relation qui s'instaure entre Levov et Zuckerman, suite d'erreurs d'interprétation de l'énigmatique surface offerte par le Suédois à l'écrivain s'aventurant à la décrypter. Doublant la tâche du narrateur-herméneute, Paule Lévy relève les correspondances les moins évidentes, inscrites dans le grain du texte, tel ce parallèle à établir entre la description par Levov du corps de sa fille nourrisson, «A body that looks quickly put on after having just been freshly ironed-no folds anywhere $(A P, 270)$ » et la peinture faite de la maison d'old Rimrock, qui, "malgré son âge vénérable, semble curieusement exempte de saillie » (43). Autre forme de résonance ou de contagion, celle qui se développe entre diégèse et récit, à la faveur d'une identification entre Zuckerman et son héros, tous deux retirés à la campagne, et affectés par la même maladie. Le relais pris par le récit tient en partie au fait que «Le Paradis des Levov était perdu d'avance puisque le narrateur enquête sur des événements révolus » (46), livrés, pour l'essentiel, dès les premières pages du roman. Plus que jamais, la vigilance du lecteur est sollicitée, surtout du fait de la structure globale de l'œuvre de Roth. Ainsi que Paule Lévy le souligne, chaque fois que l'occasion s'en présente, «l'œuvre de Roth [...] se comprend et se lit dans sa continuité; elle autorise des lectures d'amont en aval comme d'aval en amont » (46). De fait, Paule Lévy introduit un réseau de références serré, qui vient éclaircir le fonctionnement de l'œuvre et sa portée, tout en permettant une mise en contexte historique et plus largement culturel; par exemple, l'indication bibliographique donnée en note, renvoyant à la définition proposée par Sacvan Bercovich de la jérémiade américaine, dont Zuckerman « retrouve parfois les accents » (51), rappelle qu'elle a «joué un rôle majeur dans la constitution du mythe de l'Amérique » (ma traduction). De même, Paule Lévy avance une interprétation fort convaincante de la mystérieuse formule de Levov, "'Basketball was never like this, Skip' $(A P, 19)$. Seuls existent les paradis reconstruits à partir de souvenir partiels et partiaux : re-membered. La pastorale ne saurait s'écrire que sur le mode de la fable ou sur celui de l'élégie » (52). À la fois utopie et régression, la pastorale pour Roth est "tentative dérisoire de se soustraire non seulement aux violences de l'histoire, mais également (comme le suggère la présence massive et incontournable du corps dans tout le roman) aux limites inhérentes à toute vie humaine » (54). C'est donc là un roman de la dépossession en même temps que de la 
pastorale, est-il conclu à l'issue de la première partie, qui introduit la seconde : "Le mythe pulvérisé : l'hybride, le pluriel et l'impur » (55).

Là encore, Paule Lévy sait trouver dans ce roman pourtant déjà tant analysé les citations jusque-là peu repérées qui viennent étayer son argumentation : ainsi le point de vue de Roth sur le rôle des jeunes dans le mouvement protestataire des années 60 semble relayé par Jerry $(A P, 69)$, qui voit en Merry l'emblème de cette génération : «a privileged kid from paradise» (56). S'attachant ici à l'énigmatique personnage de Merry, après l'étude consacrée à son père en première partie, Paule Lévy, citant « $\mathrm{Au}$ delà des explications, le roman ", entretien accordé à Alain Finkielkraut en 2000, éclaire son lecteur quant aux intentions de Roth: «opposer Seymour Levov à une Némésis à laquelle on imaginerait qu'il pourrait absolument résister " (57). Aussi l'inversion estelle bien repérée dans la visée de la narration, sous l'effet de l'acte terroriste perpétré par Merry : il ne s'agit plus pour Zuckerman de «célébrer avec lyrisme le mythe du Suédois [...] mais de relater par le menu la descente aux enfers de son personnage [...] et d'illustrer la fin de l'idylle pastorale » (60) - fin d'une idylle qui débute par le réveil des attitudes antisémites après l'explosion, que souligne l'auteure au profit des lecteurs moins attentifs $(62),(A P, 168)$. Rappelées aussi, les limites du narrateur, « dont le point de vue, volontiers simplificateur, ne cesse de fluctuer » (66), ce qui déstabilise encore un peu plus l'interprétation. C'est indirectement que la lecture est orientée, par exemple par le biais des réseaux lexicaux tissés tout au long du roman, tel celui de la hideur, venant traduire les «mutations» qui «affectent la famille Levov, leur entreprise, ou la nation dans son ensemble » (64). Le passage du grotesque à l'abject, relevé par Paule Lévy, laisse entendre la dimension psychologique complexe du drame qui a frappé Seymour et les siens, les enjeux inconscients intrinsèquement liés au factuel. Le fin travail des champs lexicaux avertit également le lecteur de la «lente destruction " à venir (67), plongeant l'ensemble du roman dans une atmosphère de fatalité: "À l'idéal pastoral s'oppose dès le départ, et de façon particulièrement insistante, une thématique de la division et de la transgression » (67). Sont analysés divers " croisements ironiques ", d'où la troisième sous-partie du II tire son titre, et qui sont présentés comme fondement de tout grand récit: «Au commencement étaient l'hybride, le pluriel et l'impur. Tel pourrait être selon Philip Roth l'énoncé inaugural de tout récit fondateur " (67-68). Paule Lévy souligne d'ailleurs que Dawn s'intéresse au croisement des espèces (69). Quant à Merry, "produit d'un croisement hasardeux " (69), elle semble incarner le dévoiement imprévisible du projet doublement transgressif de ses géniteurs, ainsi que peut le suggérer, sous forme condensée et d'autant plus agressive, le discours de Jerry : "She's post-Catholic, he's post-Jewish, together they're going to go out there to Old Rimrock to raise post-toasties. Instead they get that fucking kid $(A P, 73) »(69)$. Paule Lévy s'attache toutefois à montrer au lecteur en quoi « [l]'avenir de cette enfant » était « inscrit d'avance. Levov entend la façonner avec le même soi, la même passion qu'il apporte à son métier " (71-72). En effet, l'auteure rappelle le rêve de Seymour quand il découvre la maison et imagine déjà sa fillette sur la balançoire. Sont également fournies au lecteur nombre de références venant compléter l'analyse en l'affinant, tel l'article sur le rôle de la nourriture dans American Pastoral, mentionné à la note 1 de la page 73. Le roman de Roth, et son œuvre tout entière, sont volontiers resitués dans la littérature juive américaine, par exemple à l'occasion de cette comparaison à Saul Bellow, qui avec Mr. Sammler's Planet met en cause « tous les courants idéologiques dont se tissent les nébuleuses sixties. Or la vraie cible de Philip Roth, c'est l'étroitesse d'esprit » (74). 
6 Après la chute, c'est l'interminable temps des interrogations que la troisième partie s'attache à analyser. Intitulée "Perspectives", elle pose la fonction révélatrice de l'explosion, mais une révélation dont l'objet, déformé, aurait été rendu méconnaissable : « Tout se passe en fait comme si chaque personnage évoluait dans une galerie de glaces où il confronterait, dans la stupeur et l'horreur mêlées, des doubles inversés ou grotesques de lui-même qu'il ne saurait reconnaître " (85). Paule Lévy montre bien comment les affres de la question "pourquoi ", que Seymour découvre tout juste, se traduisent dans la troisième partie du roman par un travail de la structure temporelle («l'usage du présent simple - tout à fait inhabituel en anglais - et l'abondance des répétitions " 88), pour donner au lecteur l'impression d'un «temps non seulement figé mais aussi parfois résolument mortifère dont il n'y aurait strictement plus rien à attendre » (91). L'auteure fait apparaître l'ambiguïté attachée, dans American Pastoral, à l'exigence de savoir, qui à la fois incite Levov à s'ouvrir à d'autres points de vue en écoutant son entourage mais aussi à harceler Merry de questions quand il la suit dans son bouge, la « somm[ant] [...] de se justifier » (93). Si la "quête du sens invariablement frustrée [...] constitue [...] l'essentiel du roman » (88), c'est que, comme le démontre Paule Lévy, "Tout le roman va en fait s'employer à exhumer, puis à donner forme, voix et sens aux mots qui n'ont pu être trouvés. » (96). Ce « discours cribl[é] de silences" (96) est analysé avec grande finesse, ses «pronoms démonstratifs privés de référent", "la prolifération des indéfinis", tel le terme «thing " répété à l'envi pour suggérer l'innommable que Seymour n'ose plus attendre $(A P, 176),(97)$. Or l'auteure voit en l'impossibilité à dire le moteur même du récit, dont «il rythme les ellipses temporelles et génère l'étonnante prolifération des filtres narratifs » (97).

L'analyse touche à son terme en mettant en lumière les "grandes préoccupations rothiennes : la hantise de la mort, le rapport ambigu entre l'art et la vie, la part d'échec inhérente à toute tentative d'interprétation et de transcription du réel » (100). Inspirée par la réalité, la fiction se trouve légitimée dans son inventivité la plus folle. L'erreur et le décalage assumés se font non seulement sources de récit, mais sources de vie. «De l'inévitable marge d'erreur inhérente à toute transcription, Philip Roth fait donc un espace de liberté " (102). C'est que, comme le propose Paule Lévy, il s'agit, dans l'exercice ludique de l'imagination, et «Par-delà le 'plaisir du texte' [...] d'apprivoiser l'aliénation, la terreur et la perte, [...] de déplacer à volonté les miroirs réfléchissants, de faire jouer les prismes et varier les distances pour laisser entrevoir l'abîme sans toucher le fond...» (105). Cette partie s'achève sur une exploration plus fouillée de la «paradoxale proximité » entre Zuckerman et «Levov, ce géant blond si limité qu'il semble être en tout point son antithèse » (107).

Or, ce qui finalement rapproche tous les personnages du roman, c'est la métaphore structurante du bégaiement, qui «dit l'opacité désarmante du réel [...] infiniment instable [...] toujours récalcitrant à l'emprise des mots » (108). Aussi, le passage cité par Paule Lévy de The Counterlife vient-il éclairer la structure du roman et du monde telles que les perçoit Roth: "The burden isn't either/or, consciously choosing from possibilities equally difficult and regrettable-it's and/and/and/and/and as well. Life is and: the accidental and the immutable, the elusive and the graspable, the bizarre and the predictable, the actual and the potential, all the multiplying realities, entangled, overlapping, colliding, conjoined-plus the multiplying illusions $(T C, 351) »(108)$. 
9 En guise de conclusion, au titre très rothien « Surtout ne pas conclure ", est rappelée la force créatrice de la réinvention de l'histoire de Levov par Zuckerman : "s'identifier à ce héros mythique d'une époque révolue, [...] c'est se soustraire au diktat exsangue des 'faits' ; c'est se jouer du corps qui aura de toute façon le dernier mot ; [...] c'est exprimer des sentiments mêlés à l'encontre d'une Amérique à la fois haïe, moquée et passionnément aimée [...]. C'est pouvoir exister à l'égard de soi-même et d'autrui. C'est réinventer sa vie ; la vie » (110).

10 Sans aplanir les difficultés que présente American Pastoral, mais au contraire en les faisant ressortir tout en montrant leur légitimité, Paule Lévy permet au lecteur de mieux apprécier encore les subtilités de ce roman de Roth et, plus largement, les pouvoirs de la fiction. Le lecteur curieux d'en savoir plus sur ce roman retirera en outre de l'étude de Paule Lévy un précieux viatique pour aborder la fiction contemporaine.

INDEX

Thèmes : Recensions

\section{AUTEUR}

ANNE-LAURE TISSUT

Université de Rouen 